Title : will be set by the publisher

Editors : will be set by the publisher

EAS Publications Series, Vol. ?, 2019

\title{
STATUS AND PROSPECTS OF THE DMTPC DIRECTIONAL DARK MATTER EXPERIMENT
}

\author{
Jocelyn Monroe for the DMTPC Collaboration ${ }^{1}$
}

\begin{abstract}
The DMTPC directional dark matter detection experiment is a low-pressure $\mathrm{CF} 4$ gas time projection chamber, instrumented with charge and scintillation photon readout. This detector design strategy emphasizes reconstruction of WIMP-induced nuclear recoil tracks, in order to determine the direction of incident dark matter particles. Directional detection has the potential to make the definitive observation of dark matter using the unique angular signature of the dark matter wind, which is distinct from all known backgrounds. This talk will briefly review the experimental technique and current status of DMTPC.
\end{abstract}

\section{Introduction}

Despite strong astrophysical evidence that dark matter comprises approximately $23 \%$ of the universe (Spergel et al. 2007), the nature of this dark matter remains largely unknown. Weakly interacting massive particles (WIMPs) are a favored dark matter candidate. Direct WIMP detection experiments search for the interaction of WIMPs with a nucleus in the detector, resulting in low-energy nuclear recoils. Most experiments seek to detect the kinetic energy deposited by the recoiling nucleus; a handful of recent efforts, including this work, also seek to detect the direction of the nuclear recoil, and in this way, infer the direction of incoming WIMPs (Ahlen et al. 2010).

The arrival direction of WIMPs is predicted to peak in the direction opposite to the earth's motion around the galactic center, and have a time-varying asymmetry in the forward vs. backward direction distribution because of the Earth's rotation (Spergel 1988). No background is expected to have the same angular distribution and modulation as a dark-matter induced signal, and therefore directional detection offers the potential for unambiguous observation of dark matter (Green et al. 2007). This paper discusses recent progress from the Dark Matter

${ }^{1}$ Department of Physics, Royal Holloway University of London 
Title : will be set by the publisher

Time Projection Chamber (DMTPC) collaboration toward a directional detection experiment.

\section{DMTPC Experiment}

DMTPC is a dark matter detector designed to measure the direction and energy of recoiling nuclei. The dark matter target is $\mathrm{CF}_{4}$, selected for the relatively large predicted axial-vector coupling for fluorine, allowing sensitivity to spin-dependent WIMP interactions (Lewin \& Smith 1996) with relatively small target masses. The detector consists of two optically isolated back-to-back low-pressure time projection chambers, with a $14.6 \times 14.6 \times 19.7 \mathrm{~cm}^{3}\left(15.9 \times 15.9 \times 19.7 \mathrm{~cm}^{3}\right)$ fiducial volume for the top (bottom) TPC. The TPCs are filled with $75 \pm 0.1$ Torr of $\mathrm{CF}_{4}$ gas corresponding to a $3.3 \mathrm{~g}(2.85 \mathrm{~g})$ fiducial mass of $\mathrm{CF}_{4}(\mathrm{~F})$. In 75 Torr of $\mathrm{CF}_{4}$, a recoiling fluorine nucleus with $50 \mathrm{keV}$ kinetic energy travels approximately $1 \mathrm{~mm}$ before stopping. The cathode and ground planes of the TPC are meshes with $256 \mu \mathrm{m}$ pitch. The grounded mesh sits $0.5 \mathrm{~mm}$ from the copper anode plate. Ionization electrons from interactions in the fiducial volume drift in a uniform electric field towards the anode region where avalanche multiplication amplifies the electron signal and produces scintillation. The gas gain is approximately $4 \times 10^{4}$, measured with an ${ }^{55} \mathrm{Fe}$ calibration source. The photon/electron production ratio in the avalanche region is $0.34 \pm 0.04$, and the wavelength spectrum of the scintillation light peaks at $\sim 600 \mathrm{~nm}$ (Kaboth et al. 2008). Scintillation light produced in the amplification region is focussed by a Nikon photographic lens (f/1.2) onto an Apogee Alta U6 camera containing a $1024 \times 1024$ element Kodak 1001E CCD chip. To improve the signal-to-noise ratio, pixels are binned $4 \times 4$ prior to digitization. Example alpha and nuclear recoil tracks are shown in Fig. 1. In addition to optical readout, the charge induced on the ground mesh and anode electrodes is also digitized. For a more detailed discussion of the 10 liter detector see (Ahlen et al. 2011).

The detector is calibrated with alpha and gamma sources. Track length and energy calibrations employ ${ }^{241} \mathrm{Am}$ alpha sources at fixed locations in the top and bottom TPCs. The length calibration relies on the known horizontal separation, $2.5 \pm 0.1 \mathrm{~cm}$, of resistive separators in the amplification region. Imaging the spacers gives the length calibration of $143 \pm 3 \mu \mathrm{m} \times 143 \pm 3 \mu \mathrm{m}(156 \pm 3 \mu \mathrm{m} \times 156 \pm 3 \mu \mathrm{m})$ for each CCD pixel of the top (bottom) anode. The energy calibration analysis compares the integral light yield of segments of alpha tracks at known distances from the source, in the arbitrary digital units (ADU) of the CCD, with the SRIM simulation (Ziegler, Biersack, \& Littmark 1985) prediction for the visible energy loss in that segment. The segment length is chosen such that the SRIM prediction for the energy loss in each segment is $100-1000 \mathrm{keV}$, depending on the location and size of the segment along the alpha track. According to SRIM, at these energies, the alpha energy loss is $>97 \%$ electronic and so we are not sensitive to assumptions about the nuclear quenching in this calibration. This procedure gives the energy calibration of $9.5 \pm 0.5$ and $12.9 \pm 0.7 \mathrm{ADU} / \mathrm{keV}$ respectively for the top and bottom cameras. The system gain $(\mathrm{ADU} / \mathrm{keV})$ may vary with position and 


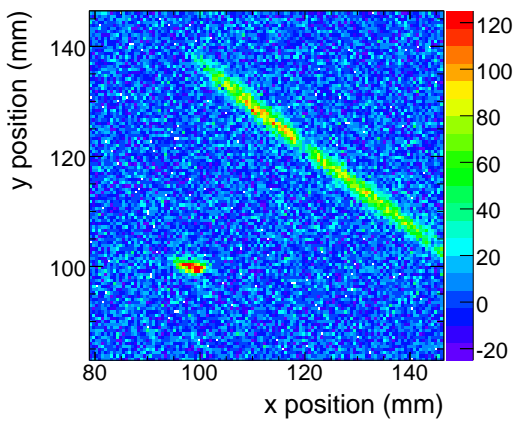

Fig. 1. A CCD image showing a sub-region of a background-subtracted event from the top camera containing a segment of an alpha track (long) and a candidate nuclear recoil (short); intensity is in units of ADU, indicated by color.

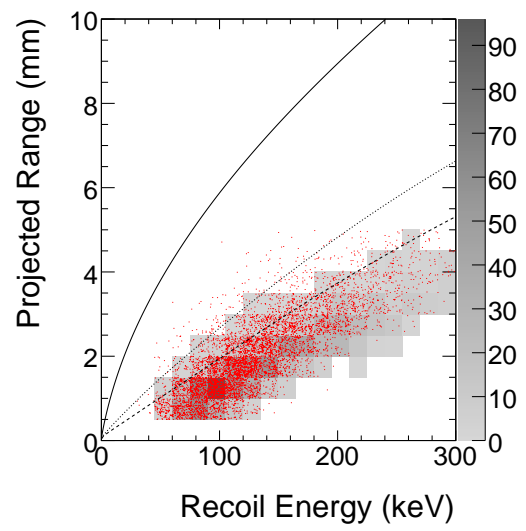

Fig. 2. Reconstructed projected range ( $\mathrm{mm})$ vs. reconstructed energy (keV) for WIMPsearch data (black points) compared with $200 \mathrm{GeV} / \mathrm{c}^{2}$ WIMP-F Monte Carlo (shaded squares) after nuclear recoil selection cuts. Lines are SRIM predictions for the maximum projected range vs. energy for helium (solid), carbon (dotted), and fluorine (dashed).

in time. The gain non-uniformity across the field of view due to local variations of the amplification is measured with a $14 \mu \mathrm{Ci}{ }^{57} \mathrm{Co}$ source, which provides uniform illumination of the field-of-view from scattered $122 \mathrm{keV}$ photons, integrated over 10,000 seconds. The measurement yields a $10 \%$ variation of the total system gain, which is included as a position-dependent correction. The stability of the gain vs. time was measured to be $1 \%$ over 24 hours using an ${ }^{241} \mathrm{Am}$ alpha source. To maintain this, the chamber is evacuated to $10 \mathrm{mTorr}$ and refilled with $\mathrm{CF}_{4}$ every 24 hours.

In the track reconstruction, raw CCD images are first background subtracted using an average of 100 dark frames (collected every 1000 exposures). Events 
Title : will be set by the publisher

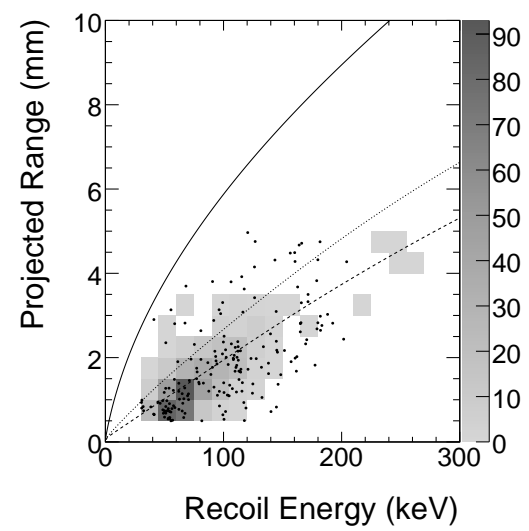

Fig. 3. Reconstructed projected range $(\mathrm{mm})$ vs. reconstructed energy $(\mathrm{keV})$ for ${ }^{252} \mathrm{Cf}$ calibration data (red points) compared with ${ }^{252} \mathrm{Cf}-\mathrm{F}$ Monte Carlo (shaded squares) after nuclear recoil selection cuts. Lines are SRIM predictions for the maximum projected range vs. energy for helium (solid), carbon (dotted), and fluorine (dashed).

which are consistent with sparking and CCD noise, such as spark-induced RBI backgrounds, are removed (Rest et al. 2002). The track finding algorithm identifies groups of five contiguous bins with at least $3.7 \sigma$ counts per bin above the image mean as clusters; 3.7 is chosen to optimize the energy reconstruction. The visible energy is the integral of the counts in a track, divided by the energy calibration constant $(\mathrm{ADU} / \mathrm{keV})$. To convert visible to nuclear recoil energy we use the $\mathrm{CF}_{4}$ quenching factor calculated in (Hitachi 2008). The projected range of a track on the amplification plane is calculated from the maximally separated pixels in the cluster, multiplied by the length calibration $(\mu \mathrm{m} / \mathrm{pixel})$. The track angle projected on the amplification plane $(\phi)$ is determined by finding the major axis angle of an ellipse with the same second moment as the pixels in the cluster. The sense of the direction is estimated from the skewness of the track light yield. The recoil energy and angle reconstruction resolution are $15 \%$ and $40^{\circ}$ at $50 \mathrm{keVee}(80 \mathrm{keV}$ nuclear recoil energy). The energy resolution is measured with alpha calibration data (Caldwell et al. 2009). The angular resolution is estimated with a Monte Carlo simulation of fluorine recoils from the ${ }^{252} \mathrm{Cf}$ calibration source. The ${ }^{252} \mathrm{Cf}-\mathrm{F}$ Monte Carlo is compared with data in Figure 2. More detail on directionality studies with this detector technology can be found in (Dujmic et al. 2008).

To study detector backgrounds, a commissioning run was taken in a surface laboratory at MIT. The background rates are summarized in Tab. 1. We are pursuing rejecting backgrounds from interactions in the CCD chip and spark-induced $\mathrm{RBI}$ by requiring coincidence of $\mathrm{CCD}$ and charge readout signals, with consistent measured event energies between the two independent channels. Preliminary results indicate $10^{-2}$ rejection from the coincidence requirement (Lopez et al. 2011). In a CCD-only analysis, these events are rejected in software, identified as having 


\begin{tabular}{cc}
\hline \hline Event Selection Cut & Rate $(\mathrm{Hz})$ \\
\hline All Tracks & 0.43 \\
Residual Bulk Images & 0.15 \\
CCD Interactions & $4.4 \times 10^{-3}$ \\
Alpha Candidates & $8.2 \times 10^{-5}$ \\
Nuclear Recoil Candidates in $80<E_{R}<200 \mathrm{keV}$ & $5.0 \times 10^{-5}$ \\
\hline \hline
\end{tabular}

Table 1. Surface run event rates $(\mathrm{Hz})$ after each background rejection cut, using CCD information only, summed over cameras.

large ADU and RMS of the pixels comprising the track (Ahlen et al. 2011). Background events inside the fiducial volume come primarily from alphas and neutrons. Alpha particles are emitted by radio-impurities in or on the materials of the detector; the majority are from the stainless steel drift cage. These are rejected as CCD edge-crossing tracks. Background neutrons come primarily from ${ }^{238} \mathrm{U}$ and ${ }^{232} \mathrm{Th}$ decays in and near the detector. The nuclear recoil candidate events remaining after all background cuts are shown in Fig. 3, compared to WIMP Monte Carlo. These are consistent with neutron-induced backgrounds (Ahlen et al. 2011); for comparison, Fig. 2 shows calibration ${ }^{252} \mathrm{Cf}$ neutron-induced recoils. There is no evidence for gamma-induced electron backgrounds; the measured upper limit on the rejection is $5.6 \times 10^{-6}$ between 40 and $200 \mathrm{keVee}$ at $90 \%$ confidence level (Lopez et al. 2011).

Using the surface commissioning data we set a limit on the spin-dependent WIMP-proton interaction cross section using the method described in (Lewin \& Smith 1996). The analysis energy window, $80-200 \mathrm{keV}$, is chosen to maximize the efficiency (estimated to be $70 \%$ at $80 \mathrm{keV}$ threshold energy). There are 105 events passing all cuts in $80<E_{\text {recoil }}<200 \mathrm{keV}$ in a 35.7 gm-day exposure, with 74 predicted neutron background events based on the surface neutron spectrum measurement in (Nakamura 2005). We set a 90\% confidence level upper limit on the spin-dependent WIMP-proton cross section of $2.0 \times 10^{-33} \mathrm{~cm}^{2}$ at $115 \mathrm{GeV} / \mathrm{c}^{2}$ WIMP mass, assuming zero expected background. Further details can be found in (Ahlen et al. 2011).

\section{Outlook and Conclusions}

We have studied the backgrounds in the DMTPC 10 liter detector prototype from a surface run, and present the first dark matter limit, $\sigma_{\chi-p}<2.0 \times 10^{-33} \mathrm{~cm}^{2}$ at $90 \%$ C.L. DMTPC is investigating the power of coincident charge and light readout to further reject backgrounds. The $10 \mathrm{~L}$ detector described here began running underground at the Waste Isolation Pilot Plant outside Carlsbad, NM in October 2010. The depth of the WIPP site is $1.6 \mathrm{~km}$ water-equivalent. The gamma, muon, and radon levels have been measured, and the neutron background has been estimated at this site (Esch 2001). Based on these, we project that underground operation will lower the neutron background to $<1$ event/year. 


\section{References}

Spergel, D. N. et al., Astrophys. J. Suppl.170, p. 377.

Ahlen, S. et al., Int. J. Mod. Phys. 25, p. 1.

Spergel, D. N., Phys. Rev. D37, p. 1353.

Green, A. M. and Morgan, B., Astropart. Phys. 27, p. 142.

Lewin, J. D. and Smith, P. F., Astropart. Phys. 6, p. 87.

Kaboth, A. et al., Nucl. Instrum. Meth. A592, p. 63.

Ahlen, S. et al., Phys. Lett. B695, p. 124.

Ziegler, J. F., Biersack, J. P. and Littmark, U., Pergamon Press, New York, 1985.

Rest, A. et al., Rev. of Sci. Instrum. 73 No. 5, p. 2028.

Hitachi, A., Rad. Phys. Chem. 77, p. 1311.

Caldwell, T. et al., arXiv:0905.2549.

Dujmic, D. et al.J. Phys. Conf. Ser. 120, p. 042030.

Lopez, J. P. et al., arXiv:1109.3501.

Nakamura, T., Nunomiya, T., Abe S., Terunuma, K. and Suzuki, H. J. of Nucl. Sci. and Tech. 42 No. 10.

Esch, E. I., Ph.D. Thesis, LA-UR-01-3974. 